\title{
Polarimetric Properties and Modeling of The Power Delay Profile in Large Hall Scenarios
}

\author{
Shiqi Cheng, Davy P. Gaillot, \\ Pierre Laly and Martine liénard \\ University of Lille I \\ IEMN - TELICE, Batiment P3 \\ Villeneuve d'Ascq, 59655 FR \\ Email: davy.gaillot@univ-lille1.fr
}

\author{
Thierry Demol \\ CITC-EuraRFID
}

165 Avenue de Bretagne, Lille, 59000 FR

Email: tdemol@citc-eurarfid.com

\author{
Emmeric Tanghe, Wout Joseph \\ and Luc Martens \\ Ghent University \\ iMinds - Department of Information Technology \\ B-9050 Ghent, BE \\ Email: emmeric.tanghe@intec.ugent.be
}

\begin{abstract}
A full-polarimetric model of the power delay profile (PDP) is proposed in a large hall scenario and validated with polarimetric measurements of a large open hall radio channel under Line-of-Sight conditions at $1.3 \mathrm{GHz}$. The measured radio channels were processed by the high-resolution parametric estimator RiMAX to estimate both the polarimetric specular multipath components (SMC) and dense multipath components (DMC). The model of the full-polarimetric distance-dependent PDP was derived from which the depolarization mechanisms are presented. In addition, it is demonstrated that the room electromagnetics theory applies to our scenario across all polarization links. The validity of the proposed model is provided by the good agreement between the polarimetric data and models. The results of this contribution highlight the fact that a complete polarimetric description of all propagation mechanisms is desired in polarimetric radio channel models.
\end{abstract}

\section{INTRODUCTION}

Over the recent years, extensive experimental studies have been performed with the aim of providing a deeper physical comprehension of the propagation mechanisms and developing physically-sound radio channel models for all types of scenarios [1]. Originally, the radio channel was commonly considered as a mere collection of Specular Multipath Components (SMC) that have well-defined discrete locations in the different radio channel dimensions (e.g., space, frequency, time, etc.). Furthermore, distributed diffuse scattering on electrically small objects and SMC with low signal-to-noise ratio (SNR) values are also included into the channel but were historically interpreted as polluting noise. Nowadays, it is understood that these effects cannot be distinguished from each other and form the basis for the Dense Multipath Components (DMC). In other words, the DMC can alternatively be interpreted as the non-coherent superposition of paths with weaker SNRs than the SMC, which still follow the specular power decay as a function of distance [2], [3]. Hence, the introduction of DMC in the physical model implies that common radio channel parameters have to be re-evaluated. Indeed, faithful models of the DMC are critical to reproduce the propagation characteristics of the radio channel in indoor scenarios such as the path loss, mean delay, root mean squared (rms) delay spread. These characteristics are used for typical coverage analysis, network optimization, localization [4], or even human exposure analysis [5].
To this end, various DMC models were developed for indoor environments [6], [7], [8], [9] from the room electromagnetics theory for diffuse scattering observed in reverberation chambers [10], [11]. In those models, the DMC is typically observed as the decaying slope of the power delay profile (PDP) [12] and characterized by its reverberation time [8]. It was shown that the energetic contribution of the DMC to the radio channel can vary between $20 \%$ and $80 \%$ for indoor or industrial scenarios [2], [13], [14], [15]. In addition, the polarization characteristics of both the SMC and DMC were investigated for an industrial environment in a previous work [16]. More recently, a distance-dependent model was reported for indoor radio channels where the PDP is described as the sum of the early SMC part of the PDP and reverberant component [17].

In this work, the model presented in [17] is deeply modified to include a full-polarimetric description of the primary SMC and DMC. Indeed, radio channel models are expected to encompass a complete polarimetric description of the physical radio link that could be used for the optimization of diversitybased wireless communications or dedicated applications. The proposed model is validated with Multiple-Input MultipleOutput (MIMO) radio channel measurements in a large hall with transmitter-receiver distance range values between $10 \mathrm{~m}$ to $45 \mathrm{~m}$. The polarization characteristics of the primary SMC and DMC are discussed for the investigated scenario. Finally, the validity of the room electromagnetics theory is assessed for the investigated scenario for all polarization channels.

The paper is organized as follows: the full-polarimetric distance-dependent model is proposed in Section II as well as the polarimetric path gain and Cross-Polar Discrimination (XPD). Before concluding, Section III presents the measurement scenario and detailed analysis about the polarimetric characteristics of the scenario.

\section{Full Polarimetric Model}

The time-varying complex impulse response of the MIMO radio channel $\mathbf{h}(\tau, t, m, n)$ can be obtained by Fourier transforming sampled versions of the frequency response $\mathbf{H}(f, t, m, n)$ measured for all Tx - Rx links:

$$
\mathbf{h}(\tau, t, m, n)=\mathcal{F}^{-1}(\mathbf{H}(f, t, m, n)),
$$




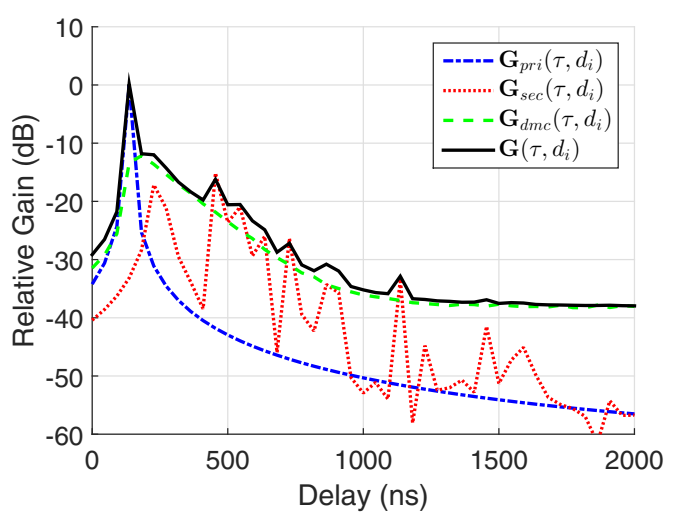

Fig. 1. Illustration of the PDP behavior in an indoor scenario for a given transmitter-receiver distance $d_{i}$. The PDP includes the primary SMC, secondary SMC, and DMC.

where $\tau$ is the time-delay, $t$ the sampled time, $f$ the sampled frequency, $m$ the $m^{t h}$ antenna of the Tx array, $n$ the $n^{t h}$ antenna of the Rx array, and $\mathcal{F}^{-1}$ the inverse Fourier operator. When the channel is not varying with time (i.e., stationary), the dependence of the radio channel on the transmitter-receiver distance $d$ can also be investigated and the PDP can be obtained from the expectation of the square magnitude of $\mathbf{h}(\tau, d, m, n)$ :

$$
\mathbf{G}(\tau, d)=\mathbf{E}\left[|\mathbf{h}(\tau, d, m, n)|^{2}\right] .
$$

The expectation operator is applied over all Tx - Rx links to remove the small scale fading.

\section{A. Polarimetric Primary SMC and DMC}

As proposed in the recent work by Steinbock et al. [17], the PDP can be split into a primary and reverberant component which both display a dependence to the distance:

$$
\mathbf{G}(\tau, d)=\mathbf{G}_{p r i}(\tau, d)+\mathbf{G}_{r e v}(\tau, d) .
$$

The primary component describes the early part of the PDP and includes the LOS (if present) and first-order reflections off the floor, ceiling, walls, and objects. In contrast, as illustrated in Fig. 1, the reverberation component describes the exponentially decaying behavior of the PDP tail and is the sum of the DMC [12] characterized by the reverberation time $\mathbf{T}$ [8], [10] and the secondary SMC:

$$
\mathbf{G}(\tau, d)=\mathbf{G}_{p r i}(\tau, d)+\mathbf{G}_{s e c}(\tau, d)+\mathbf{G}_{d m c}(\tau, d) .
$$

Here, it is assumed that the contribution of the secondary SMC to the radio channel is weak compared to the primary SMC and DMC and can be neglected. This also ensures a lower modeling complexity. Moreover, it is considered that all components can be further decomposed into a co-polar (co) and cross-polar (cross) component to take into consideration the full-polarimetric properties of the radio channel:

$$
\begin{aligned}
\mathbf{G}^{c o}(\tau, d) & =\mathbf{G}_{p r i}^{c o}(\tau, d)+\mathbf{G}_{d m c}^{c o}(\tau, d) \\
\mathbf{G}^{c r o s s}(\tau, d) & =\mathbf{G}_{p r i}^{c r o s s}(\tau, d)+\mathbf{G}_{d m c}^{c r o s s}(\tau, d) .
\end{aligned}
$$

The co-polar component is either $V V$ ( $V$ emission - $V$ reception) or $H H$ ( $H$ emission - $H$ reception), whereas the crosspolar component is either $V H$ ( $V$ emission - $H$ reception) or $H V$ ( $H$ emission - $V$ reception). This decomposition is motivated by the fact that all components will experience depolarization effects in highly-reflective environments like indoor scenarios but at different scales due to their propagation characteristics. In addition, it is believed that the path loss exponent of the primary component is polarization dependent because the environment might favor $V$ or $H$ propagation for the first-order paths. The full-polarimetric distance-dependent model for each component is given by:

$$
\begin{gathered}
\mathbf{G}_{p r i}^{c o}(\tau, d)=\chi_{p r i}(d) \mathbf{G}_{0, p r i}^{c o}\left(\frac{d_{0}}{d}\right)^{n^{c o}} \delta\left(\tau-\frac{d}{c}\right) \\
\mathbf{G}_{p r i}^{c r o s s}(\tau, d)=\left(1-\chi_{p r i}(d)\right) \mathbf{G}_{0, p r i}^{c o}\left(\frac{d_{0}}{d}\right)^{n^{c r o s s}} \delta\left(\tau-\frac{d}{c}\right) \\
\mathbf{G}_{d m c}^{c o}(\tau, d)=\chi_{d m c}(d) \mathbf{G}_{0, d m c}^{c o} e^{-\tau / \mathbf{T}^{c o}}, \tau>\frac{d}{c} \\
\mathbf{G}_{d m c}^{c r o s s}(\tau, d)=\left(1-\chi_{d m c}(d)\right) \mathbf{G}_{0, d m c}^{c o} e^{-\tau / \mathbf{T}^{\text {cross }}}, \tau>\frac{d}{c}
\end{gathered}
$$

where $\chi_{p r i}(d) \mathbf{G}_{0, p r i}^{c o}$ is the weighted gain for the primary component at reference distance $d=d_{0}$ with $\chi_{p r i}$ the distance-dependent primary polarization coefficient, $n^{c o}$ and $n^{\text {cross }}$ the path loss exponent for the co- and cross-polar primary component, and $c$ denotes the speed of light. Similarly, $\chi_{d m c}(d) \mathbf{G}_{0, d m c}^{c o}$ is the weighted gain for the DMC component at $\tau=0$ with $\chi_{d m c}$ the distance-dependent DMC polarization coefficient. $\mathbf{T}^{c o}$ and $\mathbf{T}^{\text {cross }}$ are the reverberation time for the co- and cross-polar links, respectively.

The complete polarimetric model (5)-(6) can be used to describe the radio channel characteristics (path loss, rms delay spread, etc.) whereas (7) to (10) provide a deeper understanding of the propagation mechanims of the polarimetric primary SMC and DMC.

\section{B. Polarimetric Path Gain}

From the developed model (5) to (10), the path gain averaged at distance $d$ is derived by integrating the PDP with respect to delay for each propagation mechanism and polarization state:

$$
\mathbf{G}_{Y}^{X}(d)=\int \mathbf{G}_{Y}^{X}(\tau, d) d \tau,
$$

where $Y$ is either pri or $d m c$. Consequently, the following path gain models are obtained for the co- and cross-polar components:

$$
\begin{aligned}
& \mathbf{G}^{c o}(d)=\underbrace{\chi_{p r i}(d) \mathbf{G}_{0, p r i}^{c o}\left(\frac{d_{0}}{d}\right)^{n^{c o}}}_{\mathbf{G}_{p r i}^{c o}(d)} \\
&+\underbrace{\chi_{d m c}(d) \mathbf{G}_{0, d m c}^{c o} \mathbf{T}^{c o} e^{\frac{-d}{c \mathbf{T}^{c o}}}}_{\mathbf{G}_{d m c}^{c o}(d)}
\end{aligned}
$$




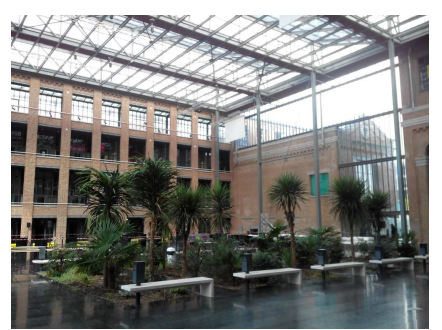

(a)

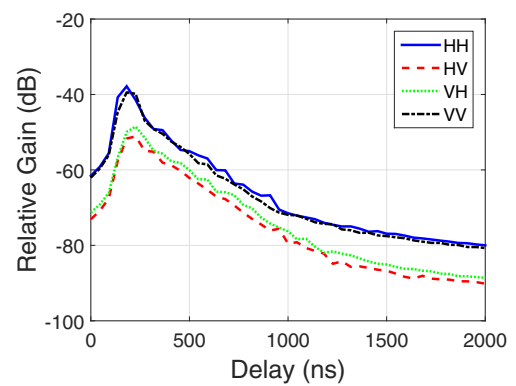

(b)

Fig. 2. (a) Picture of the EuraTechnologies Center atrium (Lille, France) from the receiving array point of view. (b) Tx (blue circles) - Rx (red cross) measurement pairs in the EuraTechnologies Center atrium. The black squares indicate the vertical brick beams visible in (a). (c) Example of measured polarimetric PDP for position 13.

$$
\begin{gathered}
\mathbf{G}^{\text {cross }}(d)=\underbrace{\left(1-\chi_{p r i}(d)\right) \mathbf{G}_{0, p r i}^{\text {co }}\left(\frac{d_{0}}{d}\right)^{n^{\text {cross }}}}_{\mathbf{G}_{p r i}^{\text {cross }}(d)}+ \\
\underbrace{\left(1-\chi_{d m c}(d)\right) \mathbf{G}_{0, d m c}^{\text {co }} \mathbf{T}^{\text {cross }} e^{\frac{-d}{c \mathbf{T}^{c t o s s}}}}_{\mathbf{G}_{d m c}^{\text {cross }}(d)} .
\end{gathered}
$$

\section{Cross-Polar Discrimination (XPD)}

The XPD is an important parameter to characterize depolarization effects in any propagation scenario. For each propagation mechanism, the distance-dependent XPD with respect to $H$ and $V$ can be related to the path gain by:

$$
\begin{aligned}
& \operatorname{XPD}_{Y}^{H}(d)(d B)=10 \log _{10}\left(\frac{\mathbf{G}_{Y}^{H H}(d)}{\mathbf{G}_{Y}^{H V}(d)}\right), \\
& \operatorname{XPD}_{Y}^{V}(d)(d B)=10 \log _{10}\left(\frac{\mathbf{G}_{Y}^{V V}(d)}{\mathbf{G}_{Y}^{V H}(d)}\right) .
\end{aligned}
$$

It follows that $\chi_{p r i}$ and $\chi_{d m c}$ can be expressed as a function of the primary SMC and DMC XPD for $H$ or $V$ by:

$$
\chi_{Y}(d)=\frac{\operatorname{XPD}_{Y}(d)}{1+\operatorname{XPD}_{Y}(d)} .
$$

(16) indicates that the polarization coefficients are strongly tied to the XPD of each propagation mechanism and naturally displays a dependence to the distance but also height. The computation of the model parameters are obtained by the set of Eqs. (13) to (16) from the measured polarimetric transfer functions and estimated SMC/DMC.

\section{VALIDATION OF THE PROPOSED MODEL}

\section{A. Measurement Scenario}

In this section, the validation of the model is discussed via the measurement and subsequent modeling of polarimetric radio channels in an indoor scenario under LOS conditions. The data was collected by a $16 \times 16$ MIMO channel sounder at $1.3 \mathrm{GHz}$ with $22 \mathrm{MHz}$ bandwidth in the atrium $(48.8 \mathrm{~m} \times 36.35 \mathrm{~m} \times 18 \mathrm{~m})$ of the EuraTechnologies Center, Lille (France). Prior being refurbished into a high-technology building, this three level-high environment was the host of a textile plant, explaining the presence of traditional brick walls, marble floor, large windows surface, and metallic structure, as depicted in Fig. 2(a). A few chairs, tables, benches, and decoration plants are located around the centre for social events such that the environment can be considered as almost empty.

The measurement campaign was performed at night without workers and visitors to ensure the time stationarity of the radio links. Figure 2(b) presents the 13 LOS Tx-Rx positions in the hall with distances ranging between $10 \mathrm{~m}$ and $45 \mathrm{~m}$. The transmitting unit was moved around the hall whereas the receiving unit was set to the same position for all measurements. A virtual uniform circular array (UCA) was used for both $\mathrm{Tx}$ and $\mathrm{Rx}$ with dual-polarized patch antennas (antenna XPD $>15 d B$ ) located at $1.6 \mathrm{~m}$ high. As an example, Fig. 2(c) presents the measured polarimetric PDP for position 13. The exponential decay of the PDP indicates the presence of DMC for all polarization links. In addition, the primary SMC can be clearly identified as the early part of the co-polar PDP but individual SMC cannot be visually distinguished from each other due to the limited bandwidth. Then, the measured channels were post-processed with a full-polarimetric version of the Maximum-Likelihood (ML) parametric estimator RiMAX [12] including the Effective Aperture Distribution Function (EADF) technique to remove the antenna contribution to the measured radio channels [18]. The mean number of estimated SMC per position was found to be around 75 . The primary SMC was built from the estimated SMC which constitute the early part of the estimated PDP. The remaining estimated SMC which form the basis of the secondary SMC (high-order reflections, diffractions, etc.) were estimated to be less $10 \%$ on average for all the positions and were neglected for this study.

\section{B. XPD Characteristics}

First, Fig. 3 presents the $\mathrm{XPD}^{H}$ and $\mathrm{XPD}^{V}$ dependence to the distance computed from the estimated primary SMC and DMC. The results show that $\mathrm{XPD}_{p r i}^{H / V}$ is much larger than $\mathrm{XPD}_{d m c}^{H / V}$. This indicates that depolarization mechanisms are mostly supported by the DMC component as also reported in [16] for a similar scenario. However, a complete depolarization of the DMC is not observed in contrast to [19] for a typical office scenario. In addition, a slow distance dependence of the depolarization process (i.e. lower XPD values) for both propagation mechanisms is observed. However, this effect is smoother for the DMC component compared to the primary one. This is attributed to the high XPD value of the LOS 


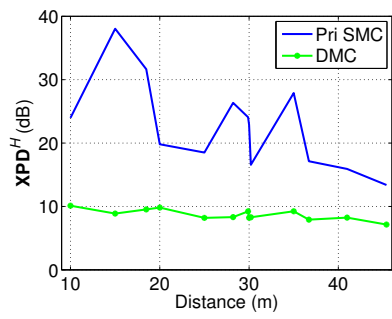

(a)

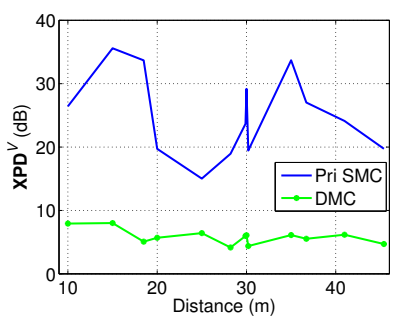

(b)
Fig. 3. $\mathrm{XPD}_{Y}^{H}$ (a) and $\mathrm{XPD}_{Y}^{V}$ (b) (in $\mathrm{dB}$ ) as a function of distance (in $\mathrm{m}$ ).

TABLE I

Propagation Model Parameters

\begin{tabular}{c|c|c|c|c|c|c|c|}
\cline { 2 - 8 } & \multicolumn{2}{|c|}{ Meas. Channels } & \multicolumn{2}{c|}{ Est. SMC } & \multicolumn{3}{|c|}{ Est. DMC } \\
\cline { 2 - 8 } & $\chi$ & $n$ & $\chi_{p r i}$ & $n_{p r i}$ & $\chi_{d m c}$ & $n_{d m c}$ & $T(n s)$ \\
\hline \hline$H H$ & 0.975 & 1.63 & 0.994 & 1.64 & 0.909 & 1.14 & 125.3 \\
\hline$H V$ & $/$ & 0.88 & $/$ & 0.95 & $/$ & 0.85 & 126.6 \\
\hline$V H$ & $/$ & 0.86 & $/$ & 0.90 & $/$ & 0.78 & 126.6 \\
\hline$V V$ & 0.957 & 1.21 & 0.971 & 1.22 & 0.853 & 1.11 & 116.1 \\
\hline
\end{tabular}

component which introduces a bias in the data and shadows the depolarization mechanisms of the first-order reflections. Finally, the scenario exhibits an XPD imbalance for the DMC which results in a more favorable propagation condition for $H$ than for $V$. Those results are in contrast with [16] where the opposite effect was reported. For that work, metallic machinery was scattered all over the floor whereas the scenario investigated in this work is almost empty. Consequently, the presence of scatterers in the same azimutal plane as the Tx-Rx arrays strongly modify the polarimetric characteristics of the channels.

\section{Path Gain Modeling}

1) Model Parameters: Table I presents the polarimetric path loss exponent obtained from the measured channels as well as the distance-dependent model parameters for the reference distance $d_{0}=10$ obtained from the estimated primary SMC and DMC. The polarization coefficient was averaged over all positions as it was observed to be weakly dependent to the distance. First, the measured channel path loss exponents are ranging between 1.2 and 1.6 for the co-polar links and are around 0.9 for the cross polar. Those exponents are in agreement with values reported in [1], [16], [20]. For the co-polar links, it is observed that $n^{H H}$ is larger than $n^{V V}$ indicating that $V V$ is the most favorable co-polar propagation link. However, the joint analysis of $n_{s m c}$ and $n_{d m c}$ reveals that the measured $n$ is solely representative of the SMC components since respective values are close. This is attributed to the low path loss exponent for the DMC despite strong reverberation values as it will be discussed later. In contrast, the measured $n^{H V}$ and $n^{V H}$ are about 0.9 and close to $n_{s m c}$ and $n_{d m c}$ for the same cross-polar links, thus indicating a high degree of correlation between the SMC and DMC loss mechanisms.
2) Model vs. Measurements: Fig. 4 presents the complete distance-dependent polarimetric model and measurements of the path gain, which includes both the primary SMC and DMC. Table II presents the root-mean-square error (RMSE) values between the path gain deduced from the primary SMC/DMC models and measurements (in $\mathrm{dB}$ ) for each polarization link. A good agreement is obtained between the models and measured data over the whole investigated distance range. Moreover, the RMSE values demonstrate the goodness-of-fit of the model to predict the trends and characteristics for each propagation mechanism and polarization link. It is observed larger RMSE values for the cross-polar primary SMC than for the co-polar links. Conversely, smaller RMSE values are obtained for the cross-polar DMC than for the cross-polar links. This can be related to the contribution of each propagation mechanism to the radio channel. For the co-polar links, the primary SMC exhibits high SNRs relatively to the DMC and, therefore, its estimate is subject to smaller error variance. For the cross-polar links, the DMC exhibits high SNRs with respect to the primary SMC such that its estimate is now subject to smaller error variance. Also, one simply observes a small slope mismatch for the DMC attributed to the constant polarization coefficient across the whole distance range as also shown in Table II. As discussed earlier for the primary SMC and DMC path gain models, a better agreement is reached for the cross-polar links compared to the co-polar links.

TABLE II RMSE $[d B]$ OF PATH GaINS

\begin{tabular}{|c|c|c|c|}
\cline { 2 - 4 } \multicolumn{1}{c|}{} & $\mathbf{G}_{\text {pri }}$ & $\mathbf{G}_{d m c}$ & $\mathbf{G}$ \\
\hline \hline$H H$ & 4.05 & 2.79 & 3.12 \\
\hline$H V$ & 7.77 & 1.42 & 1.6 \\
\hline$V H$ & 6.27 & 0.78 & 1.08 \\
\hline$V V$ & 2.93 & 2.47 & 2.37 \\
\hline
\end{tabular}

For distances lower than $10 \mathrm{~m}$ (see Fig. 4), the path gain is rapidly attenuated due to the power law behavior of the primary SMC which dominates the DMC. This region was experimentally characterized in [17]. However, for distances greater than $10 \mathrm{~m}$ (this work), the path gain is bound to the exponential decay of the DMC, which now overcomes the primary SMC. From this analysis, the cross-polar radio channels could solely be described by their reverberation time $\mathbf{T}^{\text {cross }}$ and weighted gain $\left(1-\chi_{d m c}\right) \mathbf{G}_{d m c}^{c o}$. It follows that when the validity of the room electromagnetics theory is assumed or ensured for a given scenario, the characterization of $\mathbf{T}$ via the estimator would be more robust with the crosspolar links. For instance, Table I presents $\mathbf{T}$ averaged for all positions. A standard deviation for $\mathbf{T}$ of $14.7 \mathrm{~ns}, 3.7$ ns, $2.7 \mathrm{~ns}$, and $5.5 \mathrm{~ns}$ was computed for $H H, H V, V H$, and $V V$, respectively, demonstrating that not only the room electromagnetics theory is validated across all polarization states but also that the estimation of $\mathbf{T}$ is more faithful for the cross-polar links. 


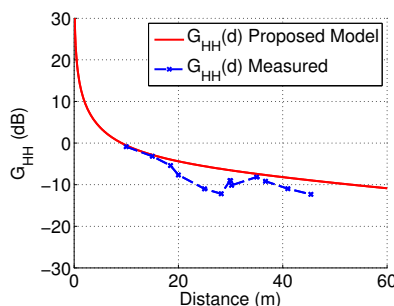

(a)

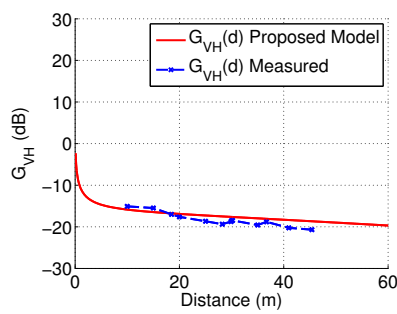

(c)

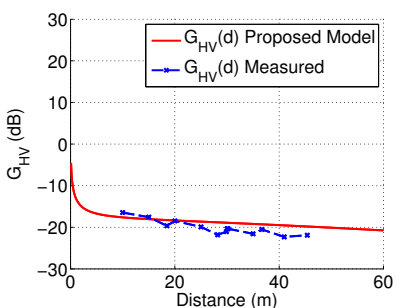

(b)

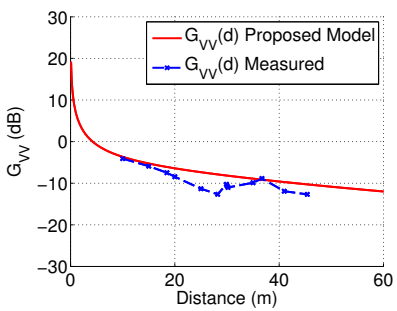

(d)
Fig. 4. $\mathbf{G}$ as a function of distance $d(\mathrm{~m})$ for (a) $H H$, (b) $H V$, (c) $V H$, and (d) $V V$ link.

\section{CONCLUSIONS}

A full-polarimetric distance-dependent model of the PDP is proposed for large hall scenarios including DMC and validated with polarimetric measurements under LOS conditions at $1.3 \mathrm{GHz}$. The measured MIMO channels were processed by RiMAX to separate the polarimetric primary SMC and DMC from which the model parameters were retrieved. The results indicate that the depolarization mechanisms are mostly supported by the DMC for the $V$ component and that the primary SMC is weak in the cross-polar links. The validity and robustness of the proposed approach is provided by the good agreement between the polarimetric data and models. Moreover, it is demonstrated that the room electromagnetics theory applies to our scenario across all polarization links. In conclusion, this contribution highlights the need to include a complete polarimetric description of both the SMC and DMC into polarimetric radio channel models such as the COST2100 [21] for instance.

\section{ACKNOWLEDGMENT}

E. Tanghe is a Post-Doctoral Fellow of the FWO-V (Research Foundation - Flanders). This work was financially supported by the INTERREG IVa project 1.1.7 WiSE and the project IAP BESTCOM, BElgian network on STochastic modelling, analysis, design and optimization of COMmunication systems. This research was also partly funded by the Fund for Scientific Research - Flanders (FWO-V, Belgium) project G.0325.11N.

\section{REFERENCES}

[1] T. Rappaport, Wireless Communications: Principles and Practice, 2nd ed. Upper Saddle River, NJ, USA: Prentice Hall PTR, 2001.
[2] J. Poutanen, J. Salmi, K. Haneda, V. Kolmonen, and P. Vainikainen, "Angular and shadowing characteristics of dense multipath components in indoor radio channels," IEEE Trans. Antennas Propag., vol. 59, no. 1, pp. 245-256, 2011.

[3] F. Mani, F. Quitin, and C. Oestges, "Directional spreads of dense multipath components in indoor environments: Experimental validation of a ray-tracing approach," IEEE Trans. Antennas Propag., vol. 60, no. 7, pp. 3389-3396, 2012.

[4] H. Liu, H. Darabi, P. Banerjee, and J. Liu, "Survey of wireless indoor positioning techniques and systems," Systems, Man, and Cybernetics, Part C: Applications and Reviews, IEEE Transactions on, vol. 37, no. 6, pp. 1067-1080, Nov 2007.

[5] A. Bamba, W. Joseph, G. Vermeeren, E. Tanghe, D. P. Gaillot, J. B. Andersen, J. d. Nielsen, M. Lienard, and L. Martens, "Validation of experimental whole-body SAR assessment method in a complex indoor environment," Bioelectromagnetics, vol. 34, no. 2, pp. 122-132, February 2013.

[6] C. Holloway, M. Cotton, and P. McKenna, "A model for predicting the power delay profile characteristics inside a room," IEEE Trans. Veh. Commun., vol. 48, no. 4, pp. 1110-1120, Jul 1999.

[7] R. Rudd, "The prediction of indoor radio channel impulse response," in Antennas and Propagation, 2007. EuCAP 2007. The Second European Conference on, Nov 2007, pp. 1-4.

[8] J. Andersen, J. Nielsen, G. Pedersen, G. Bauch, and M. Herdin, "Room electromagnetics," Antennas and Propagation Magazine, IEEE, vol. 49, no. 2, pp. 27-33, April 2007.

[9] J. Nielsen, J. Andersen, G. Pedersen, and M. Pelosi, "On polarization and frequency dependence of diffuse indoor propagation," in Vehicular Technology Conference (VTC Fall), 2011 IEEE, Sept 2011, pp. 1-5.

[10] D. A. Hill, "Electromagnetic Fields in Cavities. Deterministic and Statistical Theories”, W.-I. Press, Ed. John Wiley \& Sons, Inc., 2009.

[11] O. Delangre, "Radio channel characterization and models in reverberating rooms," Ph.D. dissertation, University of Lille1, Villeneuve d'Ascq, FR and Universit Libre de Bruxelles, Brussels, BE, 2008.

[12] A. Richter, "Estimation of radio channel parameters : Models and algorithms," Ph.D. dissertation, Technische Universität Ilmenau, Fakültat für Elektrotechnik und Informationstechnik, Ilmenau, DE, 2005.

[13] F. Quitin, C. Oestges, F. Horlin, and P. De Doncker, "Diffuse multipath component characterization for indoor MIMO channels," in Antennas and Propagation (EuCAP), 2010 Proceedings of the Fourth European Conference on, April 2010, pp. 1-5.

[14] E. Tanghe, D. P. Gaillot, M. Lienard, L. Martens, and W. Joseph, "Experimental analysis of dense multipath components in an industrial environment," IEEE Trans. Antennas Propag., vol. 62, no. 7, pp. 37973805, July 2014.

[15] J. Salmi, J. Poutanen, K. Haneda, A. Richter, V.-M. Kolmonen, P. Vainikainen, and A. Molisch, "Incorporating diffuse scattering in geometry-based stochastic MIMO channel models," in Antennas and Propagation (EuCAP), 2010 Proceedings of the Fourth European Conference on, April 2010, pp. 1-5.

[16] D. P. Gaillot, W. Tanghe, Joseph, P. Laly, V.-C. Tran, M. Liénard, and L. Martens, "Polarization properties of specular and dense multipath components in a large industrial hall," IEEE Trans. Antennas Propag., p. under revision, 2014.

[17] G. Steinbock, T. Pedersen, B. Fleury, W. Wang, and R. Raulefs, "Distance dependent model for the delay power spectrum of in-room radio channels," IEEE Trans. Antennas Propag., vol. 61, no. 8, pp. 4327-4340, Aug 2013.

[18] M. Landmann, "Limitations of experimental channel characterisation," Ph.D. dissertation, Technische Universität Ilmenau, Fakültat für Elektrotechnik und Informationstechnik, Ilmenau, DE, 2008.

[19] E. Vitucci, F. Mani, C. Oestges, and V. Degli-Esposti, "Analysis and modeling of the polarization characteristics of diffuse scattering in indoor and outdoor radio propagation," in Applied Electromagnetics and Communications (ICECom), 2013 21st International Conference on, Oct 2013, pp. 1-5.

[20] D. Xu, J. Zhang, X. Gao, P. Zhang, and Y. Wu, "Indoor office propagation measurements and path loss models at $5.25 \mathrm{GHz}$," in Vehicular Technology Conference, 2007. VTC-2007 Fall. 2007 IEEE 66th, Sept 2007, pp. 844-848.

[21] L. Liu, C. Oestges, J. Poutanen, K. Haneda, P. Vainikainen, F. Quitin, F. Tufvesson, and P. Doncker, "The COST 2100 MIMO channel model," Wireless Communications, IEEE, vol. 19, no. 6, pp. 92-99, December 2012. 